\title{
General Markowitz Optimization Problems
}

\author{
George Stoica \\ Department of Mathematical Sciences, University of New Brunswick, Saint John, Canada \\ Email: stoica@unb.ca
}

Received October 5, 2012; revised November 5, 2012; accepted November 13, 2012

\begin{abstract}
We solve two Markowitz optimization problems for the one-step financial model with a finite number of assets. In our results, the classical (inefficient) constraints are replaced by coherent measures of risk that are continuous from below. The methodology of proof requires optimization techniques based on functional analysis methods. We solve explicitly both problems in the important case of Tail Value at Risk.
\end{abstract}

Keywords: Markowitz Optimization Problems; Coherent Risk Measure; Tail Value at Risk

\section{Introduction}

We consider optimal investment for the one-step financial model with a finite number of assets. The classical Markowitz optimization problems are looking for portfolios that either maximize the expected return for a given variance threshold, or minimize the variance for a given expected return. However, using variance as a measure of risk has a serious drawback: high profits are penalized in the same way as high losses. Instead, in what follows we shall use coherent measures of risk (cf. [1]), that provide a much better quantification of risk.

In our set-up, the space of financial positions is a vector space with vector ordering $(E,+, \cdot, \leq)$. Besides the origin 0 in $E$, we distinguish a (strictly positive) reference cash stream denoted by 1 . In the space of linear price systems $E^{\prime}$, i.e., the algebraic dual of $E$, we fix a total subspace $E^{\times}$(i.e., if $f(x)=0$ for all $f \in E^{\times}$, then $x=0)$ and consider the weak ${ }^{*}$-topology on $E^{\times}$ associated to the dual pair $\left(E, E^{\times}\right)$.

A coherent measure of risk (see [2]) is a real valued mapping $\rho$ defined on $E$ which is subadditive:

$\rho(x+y) \leq \rho(x)+\rho(y)$ for $x, y \in E$, positive homogeneous: $\rho(t x)=t \rho(x)$ for $x \in E, t \geq 0$, monotonic:

$\rho(x) \leq 0$ if $x \geq 0$, and translation invariant:

$\rho(x+t \times 1)=\rho(x)-t$ for $x \in E$ and any real $t$.

The following property will be needed in our results. A coherent measure of risk $\rho$ is called continuous from below (cf. [1,3]) if $\rho\left(x_{n}\right) \searrow-1$ for any sequence $\left(x_{n}\right)_{n \geq 1}$ in $E$ satisfying $0 \leq x_{n} \nearrow 1$. Note that, if $E$ has a strong order unit, continuity from below is equivalent to the more familiar condition: $\rho\left(x_{n}\right) \searrow \rho(x)$ provided $0 \leq x_{n} \nearrow x$ (see $\left.[3,4]\right)$.

\section{Main Results}

Our first result formulates and solves in our set-up the first Markowitz problem, i.e., the so-called "agentindependent optimization problem": find portfolios that maximize the expected return for a given (measure of) risk. Particular cases have been considered in [5-8].

Theorem 1. Let $E$ be an ordered locally convex vector space, and $E^{\times}$a total Banach subspace of $E^{\prime}$. Let $x_{1}, \cdots, x_{n} \in E, c>0$ and $g \in E^{\times}$be fixed; if $\rho$ is a coherent measure of risk continuous from below, then the following optimization problem:

$$
\left\{\begin{array}{l}
\max t_{1} g\left(-x_{1}\right)+\cdots+t_{n} g\left(-x_{n}\right) \text { with } t_{1}, \cdots, t_{n} \in \mathbb{R} \\
\text { and subject to } 0 \leq \rho\left(t_{1} x_{1}+\cdots+t_{n} x_{n}\right) \leq c,
\end{array}\right.
$$

admits optimal solutions.

Proof. According to the structure theorem for coherent measures of risk (see e.g. [3], Theorem 2.1), $\rho$ admits the following representation

$$
\rho(x)=\sup _{f \in \mathcal{A}} f(-x)
$$

for some weak ${ }^{*}$-closed convex set $\mathcal{A} \subset E^{\times}$, in which all $f \in \mathcal{A}$ are positive (i.e., $f(x) \geq 0$ for $0 \leq x \in E$ ) and normalized (i.e., $f(1)=1$ ). Note that continuity from below of $\rho$ implies continuity in the order convergence topology of all $f \in \mathcal{A}$ in formula (2), see [3].

Let us define

$$
G:=\overline{\left\{\left(f\left(-x_{1}\right), \cdots, f\left(-x_{n}\right)\right), f \in \mathcal{A}\right\}},
$$

where the bar denotes closure. By the continuity from below of $\rho$ and the Krein-Šmulian theorem (see e.g. [9]), the set $\mathcal{A}$ is weak ${ }^{*}$-compact, hence $G$ is compact. Therefore, using (2), the definition of $G$, continuity from below of $\rho$ and James' theorem (see [9]), 
we obtain for any $t_{1}, \cdots, t_{n} \in \mathbb{R}$ :

$$
\rho\left(t_{1} x_{1}+\cdots+t_{n} x_{n}\right)=\max _{\left(s_{1}, \cdots, s_{n}\right) \in G}\left(t_{1} s_{1}+\cdots+t_{n} s_{n}\right) .
$$

In particular the sup in (2) is achieved, and for any $t_{1}, \cdots, t_{n} \in \mathbb{R}$ one has

$$
\begin{aligned}
& \rho\left(t_{1} x_{1}+\cdots+t_{n} x_{n}\right) \leq c \text { iff } \\
& t_{1} s_{1}+\cdots+t_{n} s_{n} \leq c \text { for any }\left(s_{1}, \cdots, s_{n}\right) \in G,
\end{aligned}
$$

where the threshold $c>0$ is given in formula (1).

As $\rho\left(t_{1} x_{1}+\cdots+t_{n} x_{n}\right) \geq 0$ for $t_{1}, \cdots, t_{n} \in \mathbb{R}$, from (2) it follows that, for some $f_{0} \in \mathcal{A}$, one has $f_{0}\left(t_{1} x_{1}+\cdots+t_{n} x_{n}\right) \geq 0$ for $t_{1}, \cdots, t_{n} \in \mathbb{R}$. Take $t_{1}= \pm 1, t_{2}=\cdots=t_{n}=0$ in the latter and, using linearity, obtain $f_{0}\left( \pm x_{1}\right) \geq 0$, i.e., $f_{0}\left(x_{1}\right)=0$. Similarly obtain $f_{0}\left(x_{2}\right)=\cdots=f_{0}\left(x_{n}\right)=0$. This means $0 \in \operatorname{int} G$, hence the following is well defined:

$$
\lambda^{*}=\inf \left\{\lambda>0: \lambda\left(g\left(-x_{1}\right), \cdots, g\left(-x_{n}\right)\right) \in G\right\} .
$$

Then the max value in (1) equals $c / \lambda^{*}$ and is achieved at every $\left(t_{1}, \cdots, t_{n}\right) \in \mathbb{R}^{n} /\{0\}$ satisfying:

$$
\begin{aligned}
& t_{1} s_{1}+\cdots+t_{n} s_{n} \geq \lambda^{*}\left(t_{1} g\left(-x_{1}\right)+\cdots+t_{n} g\left(-x_{n}\right)\right) \\
& \text { for any }\left(s_{1}, \cdots, s_{n}\right) \in G .
\end{aligned}
$$

Indeed, if $\lambda\left(g\left(-x_{1}\right), \cdots, g\left(-x_{n}\right)\right) \in G$ for some $\lambda>0$, take $\left(t_{1}, \cdots, t_{n}\right) \in \mathbb{R}^{n} /\{0\}$ satisfying

$$
t_{1} g\left(-x_{1}\right)+\cdots+t_{n} g\left(-x_{n}\right) \leq\left(t_{1} s_{1}+\cdots+t_{n} s_{n}\right) / \lambda
$$

for any $\left(s_{1}, \cdots, s_{n}\right) \in G$.

Condition (3) and definition (4) imply that $\max t_{1} g\left(-x_{1}\right)+\cdots+t_{n} g\left(-x_{n}\right)=c / \lambda^{*}$ and the $\max$ is achieved at every $\left(t_{1}, \cdots, t_{n}\right) \in \mathbb{R}^{n} /\{0\}$ satisfying condition (5). $\square$

Problem (1) is for investing a sum of money in securities; it is possible that the investor already possesses a capital with terminal value $y$, in which case minimizing the risk leads to the second Markowitz optimization problem, or "single-agent optimization problem". Alternatively, one can seek the minimum price which allows us to sell a payment order, and then compile a hedging portfolio of assets such that the risk of the entire operation will be negative or zero. Our second result formulates and solves the second Markowitz problem in our set-up.

Theorem 2. Let $E$ be an ordered locally convex vector space, and $E^{\times}$a total Banach subspace of $E^{\prime}$. Let $y, x_{1}, \cdots, x_{n} \in E$ be fixed; if $\rho$ is a coherent measure of risk continuous from below, then the following optimization problem

$$
\begin{aligned}
& \min \rho\left(y+t_{1} x_{1}+\cdots+t_{n} x_{n}\right) \text { with } t_{1}, \cdots, t_{n} \in \mathbb{R} \\
& \text { and subject to } \rho\left(t_{1} x_{1}+\cdots+t_{n} x_{n}\right) \geq 0,
\end{aligned}
$$

admits optimal solutions.

Proof. Let us denote

$$
\tilde{G}:=\overline{\left\{\left(f\left(-x_{1}\right), \cdots, f\left(-x_{n}\right), f(-y)\right), f \in \mathcal{A}\right\}} .
$$

Using a similar argument as in the proof of Theorem 1, we obtain for any $t_{1}, \cdots, t_{n} \in \mathbb{R}$ :

$$
\begin{aligned}
& \rho\left(y+t_{1} x_{1}+\cdots+t_{n} x_{n}\right) \\
& =\max _{\left(s_{1}, \cdots, s_{n}, s_{n+1}\right) \in \tilde{G}}\left(t_{1} s_{1}+\cdots+t_{n} s_{n}+s_{n+1}\right) .
\end{aligned}
$$
has

In particular, for all $u>0$ and any $t_{1}, \cdots, t_{n} \in \mathbb{R}$ one

$$
\begin{aligned}
& \rho\left(y+t_{1} x_{1}+\cdots+t_{n} x_{n}\right) \geq u \text { iff } \\
& t_{1} s_{1}+\cdots+t_{n} s_{n}+s_{n+1} \geq u \\
& \text { for some }\left(s_{1}, \cdots, s_{n}, s_{n+1}\right) \in \tilde{G} .
\end{aligned}
$$

As $\rho\left(t_{1} x_{1}+\cdots+t_{n} x_{n}\right) \geq 0$ for $t_{1}, \cdots, t_{n} \in \mathbb{R}$, using a similar argument as in the proof of Theorem 1, we obtain that the following is well defined

$$
u^{*}:=\inf \{u>0:(0, \cdots, 0, u) \in \tilde{G}\} \text {. }
$$

Then the min value in (6) is given by $u^{*}$ and is achieved at every $\left(t_{1}, \cdots, t_{n}\right) \in \mathbb{R}^{n}$ satisfying:

$$
\begin{aligned}
& t_{1} s_{1}+\cdots+t_{n} s_{n}+s_{n+1} \geq u^{*} \\
& \text { for some }\left(s_{1}, \cdots, s_{n}, s_{n+1}\right) \in \tilde{G} .
\end{aligned}
$$

Indeed, take $t_{1}, \cdots, t_{n} \in \mathbb{R}$ satisfying

$t_{1} s_{1}+\cdots+t_{n} s_{n}+s_{n+1} \geq u$ for some $\left(s_{1}, \cdots, s_{n}, s_{n+1}\right) \in \tilde{G}$. Condition (7) and definition (8) imply that $\min \rho\left(y+t_{1} x_{1}+\cdots+t_{n} x_{n}\right)=u^{*}$ and the min is achieved at every $\left(t_{1}, \cdots, t_{n}\right) \in \mathbb{R}^{n}$ satisfying condition (9). $\square$

\section{Applications}

Examples. 1) We can solve explicitly problems (1) and (6) in the important case of Tail VaR (short for Tail Value at Risk). More precisely, consider

$0<\alpha \leq 1, g \in E^{\times}$and define the Tail VaR of order $\alpha$ as the coherent measure of risk with the representation (2) in which $A=\left\{f \in E^{\times}: \alpha f \leq g\right\}$, cf. $[1,3,10]$. One can easily check that Tail VaR is continuous from below. More, Tail VaR is one of the best coherent risk measures, because is the smallest law invariant coherent risk measure that dominates the Value of Risk (cf. [3,11]). In the context of Theorem 1, we have that

$$
\left\{\begin{array}{l}
\max t_{1} g\left(-x_{1}\right)+\cdots+t_{n} g\left(-x_{n}\right) \text { with } t_{1}, \cdots, t_{n} \in \mathbb{R} \\
\text { and subject to } 0 \leq \text { Tail } \operatorname{Var}\left(t_{1} x_{1}+\cdots+t_{n} x_{n}\right) \leq c,
\end{array}\right.
$$

has the optimal solution equal to $\alpha c$. Indeed, one can easily check that $\lambda^{*}=\alpha^{-1}$ and any positive constant multiple of $(1,1, \cdots, 1)$ is an optimal solution of (1). In the context of Theorem 2, we have that 


$$
\left\{\begin{array}{l}
\text { min Tail } \operatorname{Var}\left(y+t_{1} x_{1}+\cdots+t_{n} x_{n}\right) \text { with } t_{1}, \cdots, t_{n} \in \mathbb{R} \\
\text { and subject to Tail } \operatorname{Var}\left(t_{1} x_{1}+\cdots+t_{n} x_{n}\right) \geq 0,
\end{array}\right.
$$

has the optimal solution equal to Tail $\operatorname{VaR}(y)$. Indeed, one can check that $u^{*}=\operatorname{Tail} \operatorname{VaR}(y)$ because $(0,0, \cdots, 0)$ is an optimal solution of (6). This situation occurs in problems (1) and (6) for complete models, such as Black-Scholes and Cox-Ross-Rubinstein.

2) Recall that a coherent measure of risk identifies unacceptable positions, i.e. with strictly positive risk $\rho(x)$. A good measure of the latter are the so-called relevant measures of risk: given $g \in E^{\times}$, a coherent measure of risk $\rho$ is called $g$-relevant (cf. $[1,3,10]$ ) if $x \geq 0$ and $g(x)>0$ imply $\rho(-x)>0$.

Let us consider $E=L^{\infty}(\Omega, F, P)$; we have $E^{\prime}=E^{\times}=b a(\Omega, F, P)$, the Banach space of bounded finitely additive measures on $F$ and absolutely continuous with respect to $P$. In this case, all functionals $f \in A$ (given by formula (2) above) describing a coherent measure of risk continuous from below and $g$-relevant are genuine (i.e., $\sigma$-additive) probability measures equivalent to $g$. The particular case $g=P$, i.e., $g$ represents integration with respect to $P$, has been treated in [2], Theorem 3.4, and the associated optimization problems (1) and (6) have been completely solved in $[8,12]$ (see also [4]).

The research of George Stoica was partially supported by a grant from the Natural Sciences and Engineering Research Council of Canada.

\section{REFERENCES}

[1] P. Artzner, F. Delbaen, J.-M. Eber and D. Heath, "Coherent Measures of Risk," Mathematical Finance, Vol. 9,
No. 3, 1999, pp. 203-228. doi:10.1111/1467-9965.00068

[2] F. Delbaen, "Coherent Risk Measures on General Probability Spaces," In: K. Sandmann, et al., Eds., Advances in Finance and Stochastics, Springer-Verlag, Berlin, 2002, pp. 1-37.

[3] G. Stoica, "Relevant Coherent Measures of Risk," Journal of Mathematical Economics, Vol. 42, No. 6, 2006, pp. 794-806. doi:10.1016/j.jmateco.2006.03.006

[4] L. A. Konovalov, "Coherent Risk Measures and a Limit Pass," Theory of Probability and its Applications, Vol. 54, No. 3, 2010, pp. 403-423. doi:10.1137/S0040585X97984309

[5] C. Acerbi, "Coherent Representations of Subjective Risk Aversion,” In: G. Szegö, Ed., Risk Measures for the 21st Century, Wiley, New York, 2004, pp. 147-207.

[6] R. T. Rockafellar and S. Uryasev, "Optimization of Conditional Value-at-Risk," Journal of Risk, Vol. 2, 2000, pp. 21-41.

[7] R. T. Rockafellar, S. Uryasev and M. Zabarankin, "Master Funds in Portfolio Analysis with General Deviation Measures," Journal of Banking and Finance, Vol. 30, No. 2, 2006, pp. 743-778. doi:10.1016/j.jbankfin.2005.04.004

[8] A. S. Cherny, "Equilibrium with Coherent Risk," Preprint, 2006.

[9] A. Grothendieck, "Topological Vector Spaces," Gordon and Breach, Philadelphia, 1992.

[10] S. Jaschke and U. Küchler, "Coherent Risk Measures and Good-Deal Bounds," Finance and Stochstics, Vol. 5, No. 2, 2001, pp. 181-200. doi:10.1007/PL00013530

[11] S. Kusuoka, "On Law Invariant Coherent Risk Measures," Advances in Mathematical Economics, Vol. 3, 2001, pp. 83-95.

[12] A. S. Cherny, "Pricing with Coherent Risk," Theory of Probability and Its Applications, Vol. 52, No. 3, 2008, pp. 389-415. doi:10.1137/S0040585X97983158 